\title{
ON REAL PROJECTIVE SPACES AS FINSLER MANIFOLDS
}

\section{NATHANIEL GROSSMAN}

Let $M$ be a finite-dimensional $C^{3}$ manifold supplied with a $C^{2}$ Finsler metric $d s=F(x, d x)$, which is not necessarily even in $d x$. Let $\rho$ designate the induced oriented topological metric. For any $p \in M$, the antipodal locus of $p$ is the set $A(p)=\{q \in M \mid \rho(p, q) \geqq \rho(p, r)$ for all $r \in M\}$. For example, if $M$ is a real projective space with the Riemannian metric of constant curvature $1, A(p)$ is a smooth hypersurface (in fact, a projective hyperplane) for every $p \in M$. One may ask, how close does this property come to characterizing real projective spaces among Finsler manifolds?

We prove

Theorem. Let $M$ be a finite-dimensional $C^{3}$ manifold supplied with a complete $C^{2}$ Finsler metric. Suppose $A(p)$ is a smooth hypersurface for at least one $p \in M$. Then $M$ is homeomorphic with a real projective space.

Proof. All the tools for the proof are contained in the classic paper of J. H. C. Whitehead [1], especially in $\$ 10$.

Let $p$ be a point for which $A(p)$ is a smooth hypersurface. Let exp denote the exponential map (which is of class $C^{1}$ ) of the Finsler metric at $p$. Let $\delta$ be the distance from $p$ to $A(p)$ and let $S(\delta)$ $=\left\{v \in M_{p} \mid F(p, v)=\delta\right\} . S(\delta)$ is homeomorphic with a sphere and bounds a cell.

$A(p)$ is a subset of the cut locus of $p$ and hence any $q \in A(p)$ is either hit by at least two minimal geodesics from $p$ to $q$ or is conjugate to $p$ or maybe both. In the first case, suppose $q=\exp v=\exp w, v \neq w$, and that $q$ is not conjugate to $p$. If also $q=\exp u, u \neq v, w, q$ would be a branch point for $A(p)$ contrary to $A(p)$ being a manifold. The second case cannot occur, since if only one geodesic of length $\delta$ went from $p$ to $q$, the conjugacy of $q$ would imply that $A(p)$ had codimension at least 2 at $p$. In the third case, Whitehead shows that the intersection of the cut and conjugate loci is again of codimension at least 2 .

Now, let $A^{\prime}(p) \subset S(\delta)$ be the subset of those elements carried by exp onto $A(p)$. Since $A(p)$ is closed and exp is continuous, $A^{\prime}(p)$ is closed in $S(\delta)$. Since exp is nonsingular on $A^{\prime}(p)$ and the dimensions of $S(\delta)$ and $A(p)$ coincide, $A^{\prime}(p)$ is open in $S(\delta)$. Therefore, $A^{\prime}(p)=S(\delta)$.

Received by the editors March 22, 1966. 
The interior of $S(\delta)$ is mapped by exp diffeomorphically onto $M-A(p)$ and we have just shown that $S(\delta)$ is a 2-1 covering of $A(p)$. The homeomorphism of $M$ with a real projective space is now obvious.

\section{REFERENCE}

1. J. H. C. Whitehead, On the covering of a complete space by the geodesics through a point, Ann. of Math. 36 (1935), 679-704.

Institute for Advanced Study and

University of California, Los Angeles 